
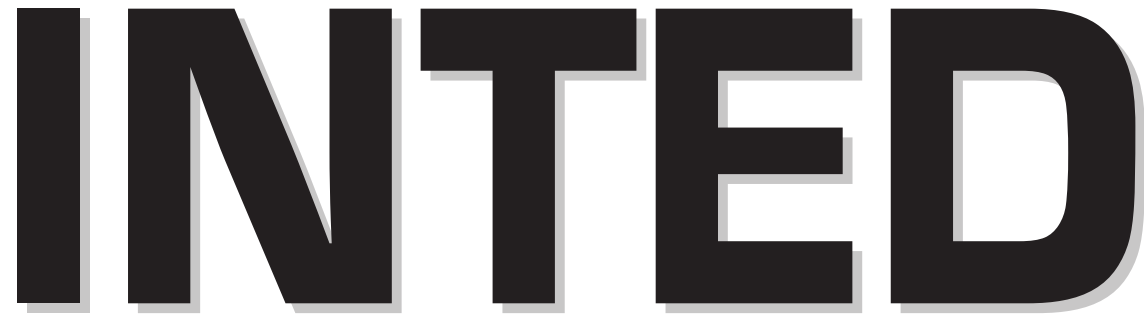

\section{7}

11th International

Technology, Education and

Development Conference

6-8 March, 2017

Valencia (Spain)

\section{CONFERENCE PROCEEDINGS}


Published by

IATED Academy

iated.org

INTED2017 Proceedings

11th International Technology, Education and Development Conference

March 6th-8th, 2017 - Valencia, Spain

\section{Edited by}

L. Gómez Chova, A. López Martínez, I. Candel Torres

IATED Academy

ISBN: 978-84-617-8491-2

ISSN: $2340-1079$

Depósito Legal: V-369-2017

Book cover designed by

J.L. Bernat

All rights reserved. Copyright (C) 2017, IATED

The papers published in these proceedings reflect the views only of the authors. The publisher cannot be held responsible for the validity or use of the information therein contained. 


\title{
THE SCIENTIFIC LEARNING ACCORDING TO VIGOTSKY
}

\author{
Enrique Arribas ${ }^{1}$, Isabel Escobar ${ }^{1}$, Teresa Franco ${ }^{2}$, Carmen Suárez ${ }^{3}$, Sarahi \\ Vidales $^{3}$, Yolanda Benítez ${ }^{4}$, Silvia Maffey ${ }^{5}$, Jesús González-Rubio ${ }^{1}$, Alberto \\ Nájera' ${ }^{1}$, Remedios Ruiz ${ }^{1}$, Augusto Beléndez ${ }^{6},{ }^{2}$. Ramirez-Vazquez ${ }^{1}$, Juan \\ Besanilla ${ }^{7}$, Cuauhtémoc Garcia-Olguin ${ }^{7}$ \\ ${ }^{1}$ Universidad de Castilla-La Mancha, Albacete (SPAIN) \\ ${ }^{2}$ Instituto Politécnico Nacional, ESIMEZ, Ciudad de México (MEXICO) \\ ${ }^{3}$ Universidad Autónoma de San Luis Potosí, Tamazunchale (MEXICO) \\ ${ }^{4}$ Universidad Nacional Autónoma de México, Cuautitlán (MEXICO) \\ ${ }^{5}$ Instituto Politécnico Nacional, Ciudad de México (MEXICO) \\ ${ }^{6}$ Universidad de Alicante, San Vicente del Raspeig (SPAIN) \\ ${ }^{7}$ ENESMAPO, Plantel 5, Tamazunchale (MEXICO)
}

\begin{abstract}
The interaction within the social environment allows for knowledge to be created by each one of us. The everyday experience of the student and the presence of the teacher are the two most important factors that make learning. Behind of each child that learns there is a child who thinks and who uses his/her language as a learning tool that permits to build higher psychological processes. The language plays a crucial role in cognitive development to obtain scientific knowledge, because if children handle words and symbols they will be able to construct scientific concepts every time they are motivated and supervised by a teacher. Vigotsky tells us that at the beginning of science you can find the word, the language.
\end{abstract}

Keywords: Active Learning, University Teaching, Conceptual Map, Electric Circuits.

\section{INTRODUCTION}

All teachers, are indebted to a Russian psychologist of Jewish origin died more than 80 years ago because of a tuberculosis. We are referring to Lev Semionovich Vigotsky (also known as Vigotsky) who lived between 1896 and 1934. He is considered one of the leading theorists of development psychology, founder of the so-called cultural-historical psychology and Neuropsychology Soviet predecessor. His work was discovered late in the decade of the 1960's by the world Western. He died at the age of 37 having published numerous works, so he is sometimes called as "the Mozart of Psychology".

\section{VIGOTSKY AND THE LANGUAGE}

According to Vigotsky, the development of human beings needs to be explained in terms of social interaction, the maturation of the student allows that his thinking acquires more and more elaborate formal structures. The Vigotskyana perception is a process that has a biochemical origin and to reach its full expansion it is necessary that culture makes its appearance, primarily through the manifestation and consolidation of the language, that is why the perception is often considered as the earliest stage in the psychological development of the child [1].

The so-called primary synthesis of perception needed other two mental processes that we can call low level to take place: the recognition and memory. In contrast with them there are other upper mental processes: intelligence, thought, analysis, synthesis, reflection, attention and abstraction (Figure 1). These seven processes are elements of the human culture that is passed from a few individuals to others. On this synthesis Vigotsky writes: "if we look at the perception of an adult, it seems that this does not consist simply of a complex synthesis of personal impressions and images of memory but is based on a complicated synthesis of the thought processes of perception processes. What we see and what we know, what we perceive and what we think, merge into a whole" [2]. 


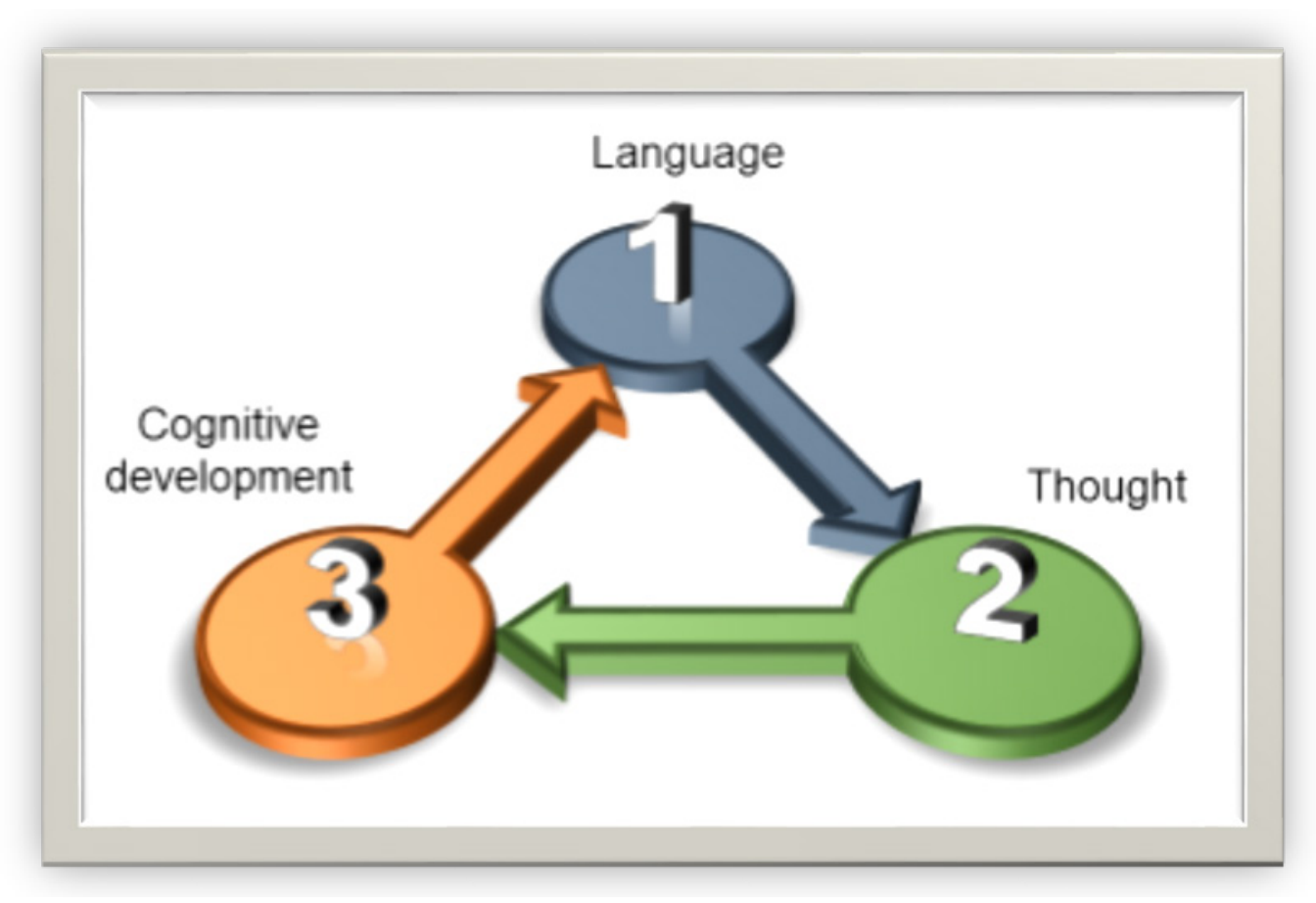

Figure 1. Language is a crucial tool in the cognitive development for scientific knowledge.

Through the use of the language acquired a dual quality, it may be subject and at the same time, object of our own learning. When a child asks, we can learn that he/she is trying to solve a problem that has raised in his/her learning, whether it is formal or not. Its demand warns us that he/she is developing a plan to solve a difficulty that he/she has, and that him by himself has not solved yet. His/her brain is in contradiction or has a doubt, ignores something that really worries him/her and that us why he/she questions.

\section{VIGOTSKY AND THE SCIENTIFIC LEARNING}

Learning is a process in continuous development, progressive and evolutionary that begins even before the school learning and that never part of zero, is based on the previously known (previous ideas). There is a continuous reorganization of the psychological processes and that is why learning is a dynamic task from a few rudiments innate to the very essence of the human being. In this dynamic process are van replaced most basic concepts by others more complex and elaborate.

But this substitution is dialectic; i.e., there is a contrast between what is known and what is learned, there is a thesis and an antithesis. This confrontation of disparate elements comes from the synthesis, a third element; i.e., a resolution, an understanding of the problem and a finding of their possible solutions. This learning process goes against tradition, is a review of what is known through an apparent contradiction between what is known and what is observed. When these processes mental have place, the quantity is transformed in quality and in the brain is produced changes in ideas interconnected (Figure 2). Learning that the child will develop is a real battle in the brain of what is already known and new coming to modify what already exists, which includes three stages.

1 Assertion. Existence of a previously acquired reality.

2 Denial. When collecting the item contrary to this reality; one questions what is known, what has already been learned.

3 The reconciliation. Most important, produces a synthesis between the positive elements of the first two steps. Generates a new reality in the mental structure of the child, which will enter a cyclic transformation in a short time. After these changes we will developing a fuller understanding and integrating. It is a process evolving, changing in time and dynamic, you will need it to revive several times throughout his life; whether it is academic, or otherwise. 


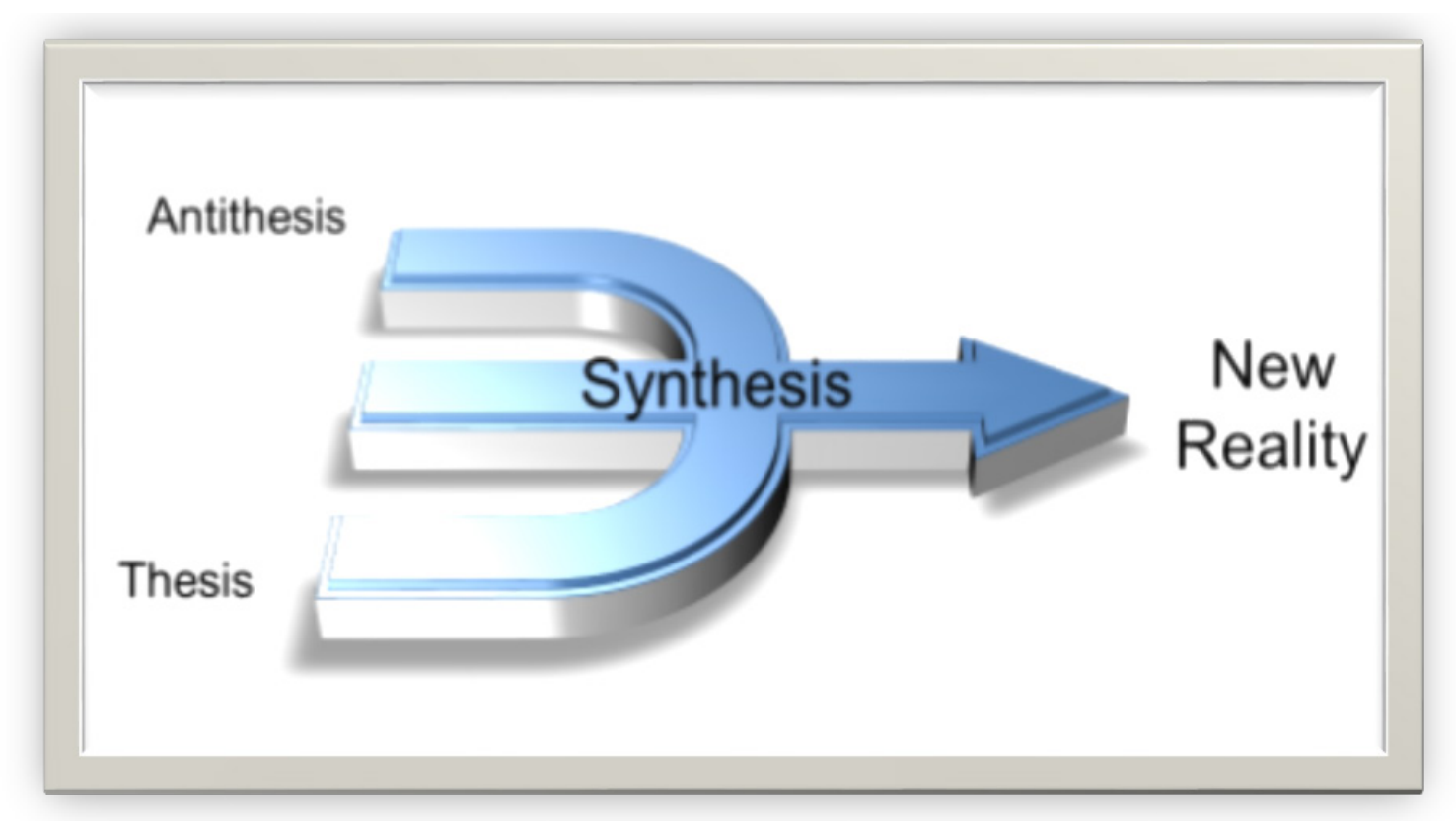

Figure 2. The learning according to Vigotsky.

The scientific knowledge learned should be incorporated into the development of children, because it is necessary that this knowledge can be applied later, in their daily experience. For this, children must be able to incorporate concepts and methods of scientific analysis into their mental schemas, this process must occur in school education. The scientific concepts are not usually spontaneous processes, they are located in the diffuse zone between the spontaneous processes and those induced in the minds by the pedagogical action. When a method of discovery learning is used, it is intended that children use their concepts spontaneously learned to deal with new problems, problems of different subjects; For this they must use tools of the scientific method (Figure 3).

The construction of the scientific concepts needs a primary verbal knowledge of them to be able to interact effectively with the teacher, so mature and superiors of the child consolidated the psychic functions. Being of higher processes advanced, the domain of scientific concepts is consolidated to participate in specific and duly planned social activities. There is a contrast between the everyday concepts and scientists. The everyday have a limited abstraction ability; Conversely, the scientific concepts needed a verbalism (in a manner of words) that will allow us to pose interrelated concepts, and good planning for the analysis of the evolution of the structures of generalization. 


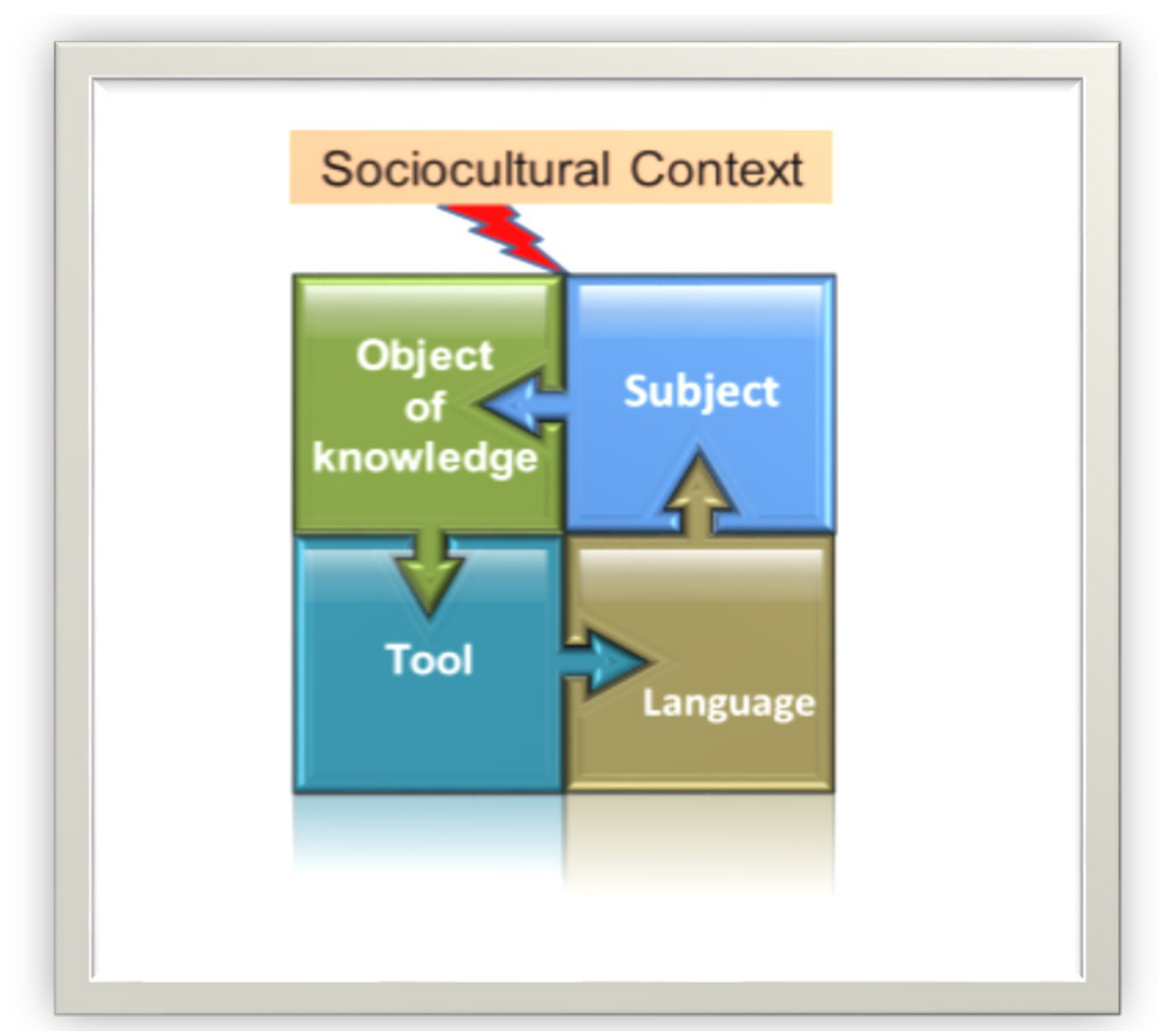

Figure 3. Relation between the subject and the object of the knowledge according to Vigotsky.

Vigotsky explains these complex intellectual operations emphasizing two aspects: their systematic nature and the presence of a conceptual system, which is the central element of this process. New ideas with the help of other upcoming concepts must be generated so that higher stages of development are activated, then the meaning of words allows you to make use of the principle of equivalence of conceptions, principle that also suffers from a genetic evolution, so it is different and specific to each stage of development. Related with this Vigotsky is that the study of the development of real concepts shows that the new phase of the development of generalizations is reached only by means of the transformation (that does not override) the foregoing objects already previously widespread in the former system and not by the generalization of isolated objects. The transition of the prejudice to the true concepts takes place through the generalization of generalized previously objects. Once apprehended, scientific concepts rearrange concepts everyday subjects, since his brain has acquired a new structure of generalization (Figure 4). 


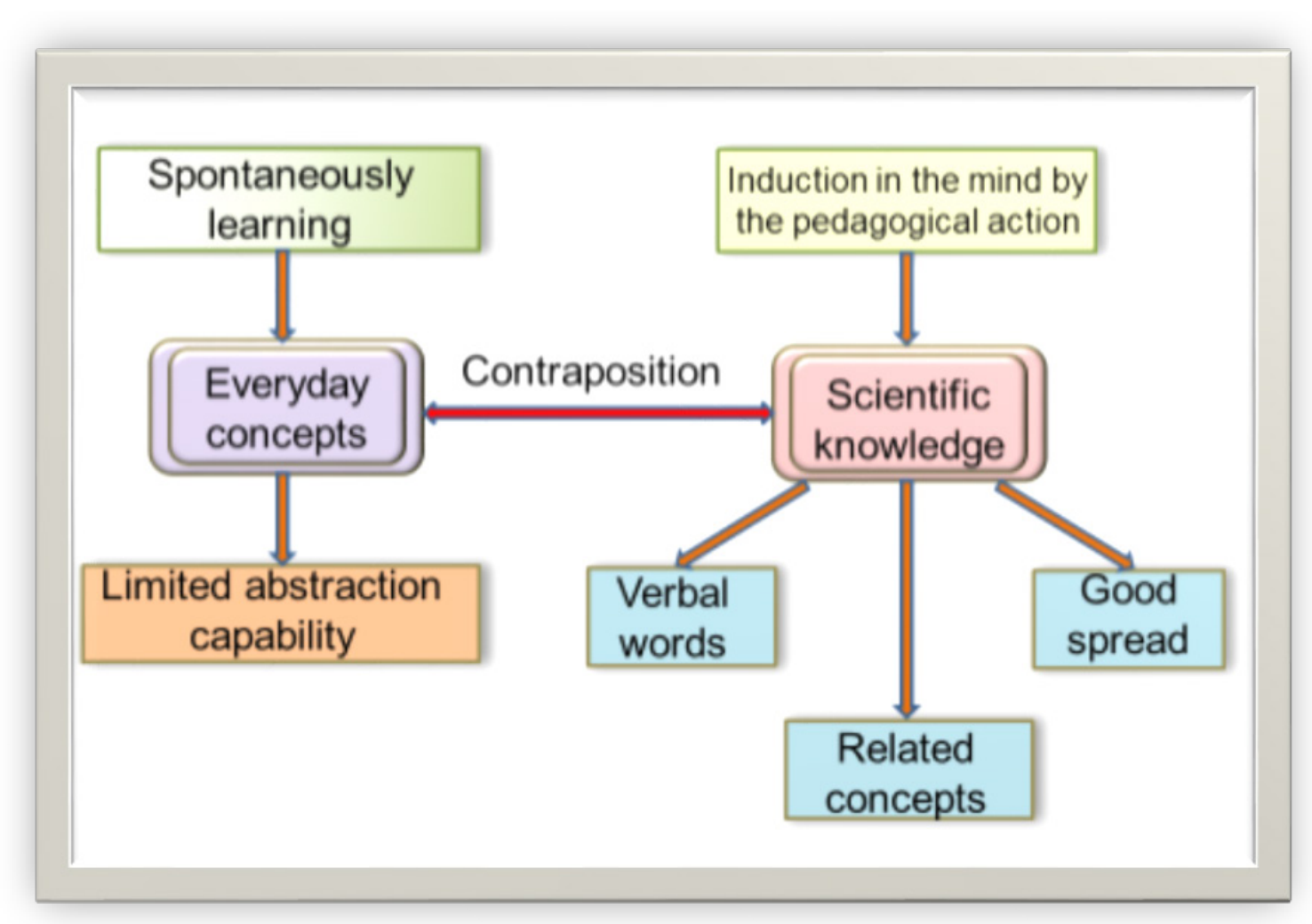

Figure 4. Contrast between the concepts of everyday life and the scientific knowledge according to Vigotsky.

\section{VIGOTSKY AND ZONE OF PROXIMAL DEVELOPMENT (ZPD)}

It is very difficult for scientific knowledge to be achieved through an autonomous process. In the first steps of scientific knowledge the assistance of a person skilled in this knowledge is needed. After the instruction, it is possible to obtain autonomy in the learning, in such a way that the development of the persons and their own learning are generating superior and dynamic psychological processes.

Vigotsky introduced the notion of zone of proximal development (ZPD) in which both the teacher and the student, act in a joint and coordinated manner in search of goals previously designed by the instructor. The teacher is no longer leading into resource; i.e., the master is a pole that the student has to get much higher learning, without the hop pole (the advance in knowledge) would be much smaller. The adult is a guide and a support, but need an active participation of the student in the process of scientific learning. When this happens it is said that both actors are involved in significant activity within a pre-defined framework of learning (Figure 6). We know that you learn more when the student receives adequate assistance by the teacher.

The ZPD has been defined as the distance between what a learner is able to perform by itself and what you can learn with the help of an expert. That space is the profit that has produced the educational action. This area gets smaller as the learning requires less support to accomplish the task. The ZPD should be conceived and planned way ambitious to achieve the main objective: the capillarity of the school within social structures to improve the teaching function. Student learning is also important, but teaching function should transcend beyond the limits of the class. With this concept you can explore the potential of learning because of the social interaction and with the expert, which allows to understand and develop more complex thoughts. 


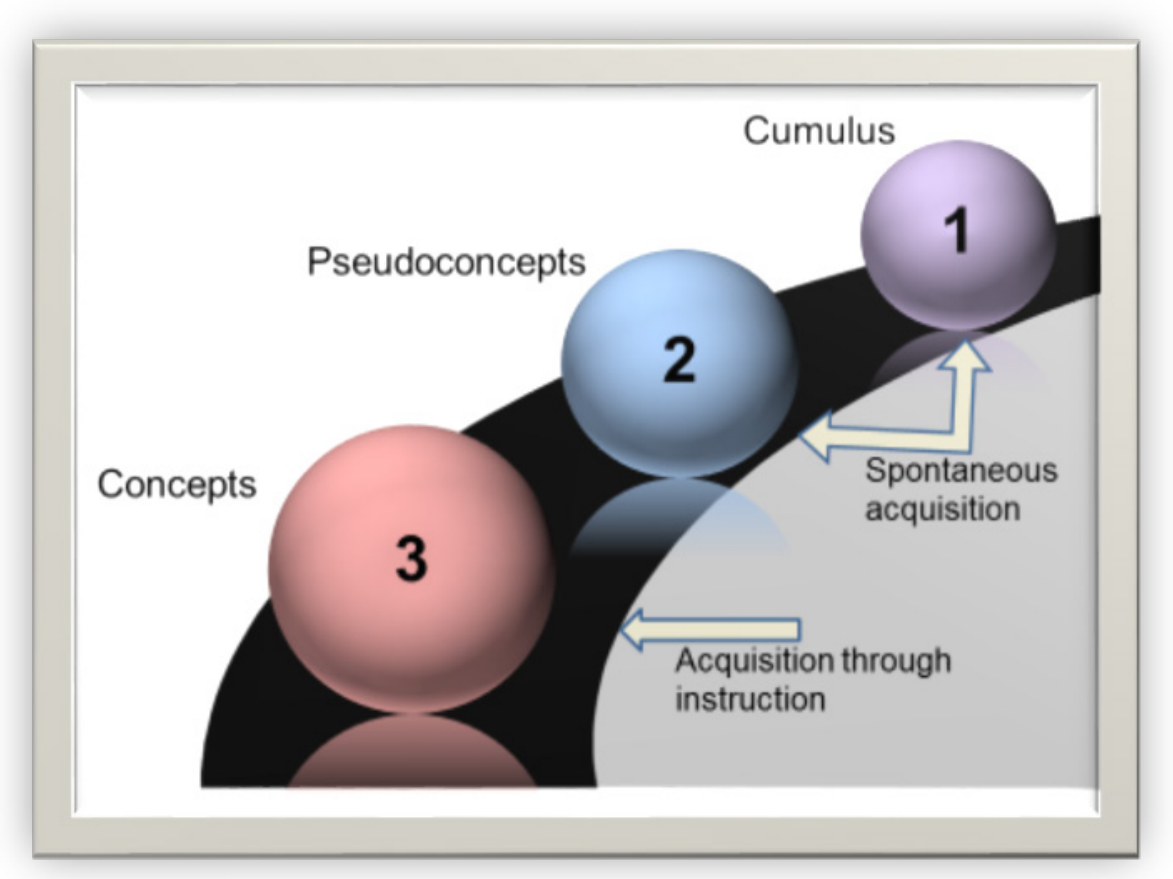

Figure 5. Acquisition of concepts.

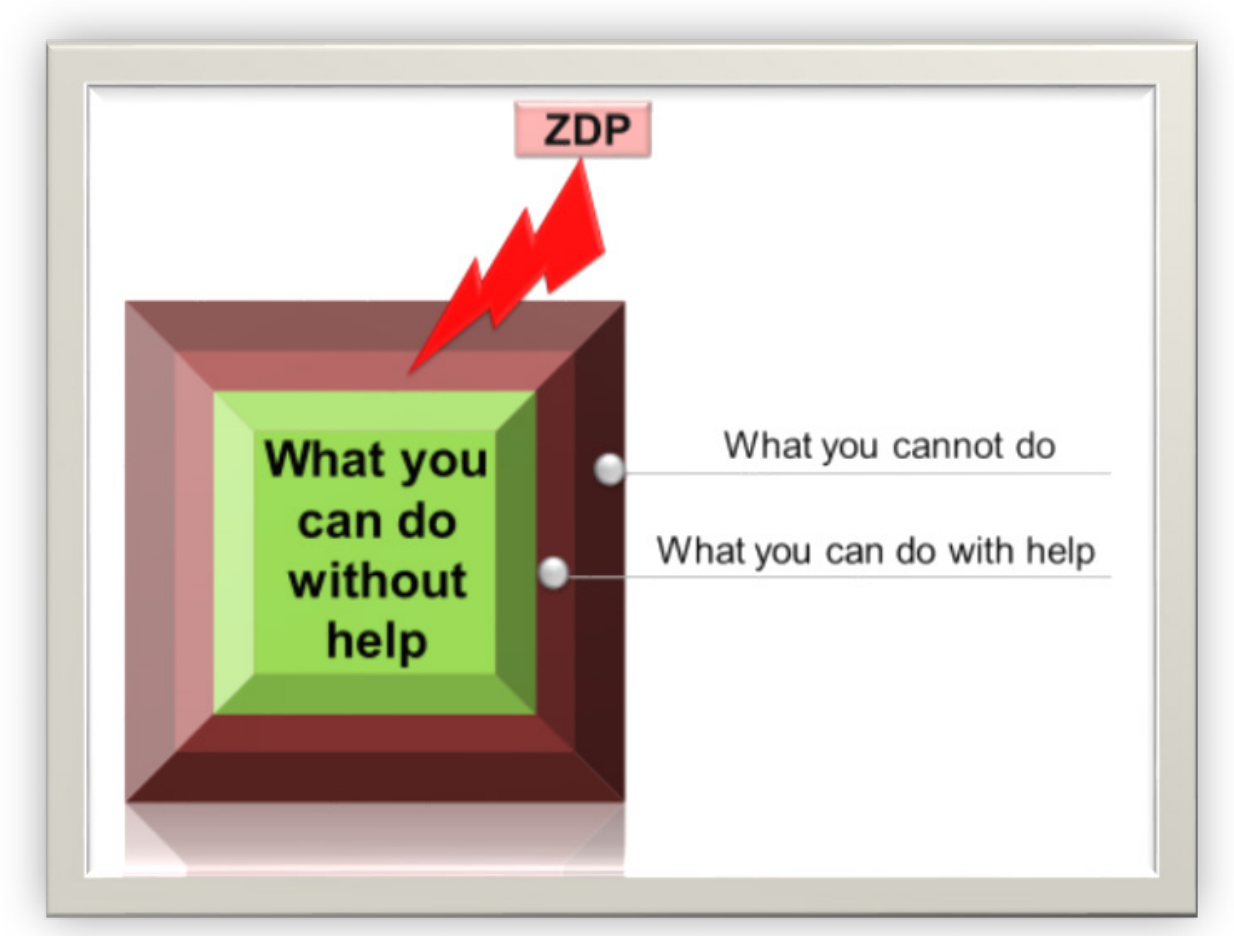

Figure 6. Zone of proximal development according to Vigotsky.

\section{VIGOTSKY AND THE CONSTRUCTIVISM}

The so-called psychological functions allow us to investigate the way in which you learn. Vigotsky discovered a complex network psychological factors involved in the various stages of learning. The psychological functions are interconnected through cross-functional factors, which will evolve dynamically according to the different stages of the evolution of people. Perception and attention allowed the emergence of symbolic function by means of which the mind uses symbols to represent a thing or an idea, for example, the concept of vector field or the symbol representing the linear acceleration. 
Learning and generating symbols (i.e. ideas) is a mental faculty that acquires the apprentice in his first approximations to the understanding of nature and its functioning, understanding the relationship between the symbol representing another object or idea and the object itself. In any process of learning symbols play an important role, along with the written and spoken language.

Perception arises from the attention, which is purely reflex action, and enables to acquire the existence and feeling of the things present and palpable. It arises from the creation of a world of its own by the apprentice, are appearing items where before there was only sensations; gradually, this is goes adapting to its environment and turning in symbols easily manageable for it, its environment is goes padded of objects, and symbols that arise of his interaction with the medium that you surround. Memory is an evolution of perception, an evolution that allows better performance, in turn, of perception. With the appearance of the memory, the perception and the care leave of be mechanisms primitive for work as functions modified by the effect of the context sociocultural.

According to Vigotsky, scientific knowledge has two notable aspects; on the one hand, all concept scientific by very high that is its grade of abstraction, has always a sediment of the reality concrete and by another, all concept scientific encloses an abstraction primary; i.e., abstraction and reality complementary and need to build the scientific knowledge. Vigotsky tells us that at the beginning of the science is the word; the language, any Word can be a theory, an inclusion of an isolated phenomenon within a category, that of similar phenomena.

All this clearly tells us that there is a priority of language, of words, of the terminology on the experiments, on the empirical facts. Those criteria conceptual are above the observations made. The observation is no longer neutral, there is that which aims to understand, or not, from a theory. Modern science has lost which is believed to be its engine: empiricism. Now the driving force is the generalization of the theories, the unification of knowledge from relatively distant fields together, the integration of heterogeneous, the inclusion of the disparate within the framework of the totality of scientific knowledge. In this hierarchical power struggle will be scientific knowledge which will achieve supremacy.

At the beginning there is a real discovery that goes beyond the border of the hitherto known, reality previously acquired, of the thesis. Occurs the negation of what exists, is in question the previously established paradigm, comes the antithesis. This overflow makes ideas to spread to adjacent fields, as when a reservoir is full. This spread makes new ideas to change the structures already created and at the same time are modified in a way completely dynamic. In these confrontations of ideas, it is germinating synthesis, the reconciliation of the thesis with the antithesis, there is resolution of the generated problem and possible solutions are obtained. This new reality, synthesis of two opposing elements, is broader and more inclusive, because it has the parties positive of the two visions. To its time, that synthesis modifies the own structure of the knowledge science. Consider just the theory of special relativity of Einstein and his constancy of the velocity of light in any reference system. Synthesis-generated idea is changing increasingly goes more spreading from its initial concept, the ramifications and connections are expanding and can even form part of the philosophical system as a universal principle or, even, an ideology.

This evolution Vigotsky called it the swollen idea, like a frog that turns cow. If it continues to evolve, it will burst like a soap bubble. In the acquisition of scientific knowledge concepts as tools are used to get the facts, but as we are continuously checking its validity, as at all times we are confronting the thesis with the antithesis, you are modifying and only survive the useful, the best that pass all the verification tests. It is an evolution of the cognitive in the field scientific, not is it logical of which arise them ideas, it is the own movement towards the ideas which makes that arise. It is a kind of spontaneous feedback.

Scientific knowledge tends to be in the debate on learning and development. There is a deep division between those who argue that these concepts already are established of way priori and which, in counterpart claim that then concepts are building of a dynamic way. According to these, each new scientific concept expands capabilities to generate a new one which arises from the already established.

The modern scientist, according to Vigotsky, may believe that it can be placed in front of the nature and master it or control it [3]. Personal development is a cultural construction, which is achieved through shared social activities. The psychological processes that are generated in this interaction are called artifacts, which allow scientist to dive in the scientific knowledge to provide necessary to share what symbolism. These artifacts may be primary, secondary or tertiary [4] depending on the degree of depth reached and the mental processes involved. Is must exit of the prehistory of the learning [5] to 
enter in a new stage in which the previous cognitive structure that the students already have is taken into account, trying to eradicate them errors conceptual pre-existing. It is once again the fight antithesis, leading to a synthesis. Scientific knowledge is not only received, you not only learn, but is transformed in the process of learning and at the same time, it also transforms the subject who receives it. It is a dynamic process, constantly changing and that is the key that allows the advancement in the understanding of nature and their operating mechanisms.

\section{REFERENCES}

[1] E. García, Pedagogía constructivista y competencias. Ciudad de México: Trillas, 2011.

[2] L. S. Vigotsky, Desarrollo de las funciones psíquicas superiores en la edad de transición, Obras escogidas. Madrid: Visor, 1982.

[3] J. Wertsch, El papel de la racionalidad abstracta en la imagen vigotskyana de la mente. Buenos Aires: Paidós, 2000.

[4] M. Cole, Psicología Cultural. Una disciplina del pasado y del futuro. Madrid: Morata, 1999.

[5] L. S. Vigotsky, Aprendizaje y desarrollo intelectual en la edad escolar. Madrid: Akal, 1973. 